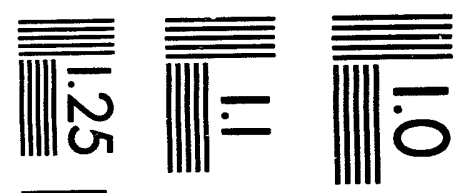

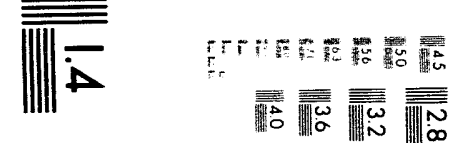

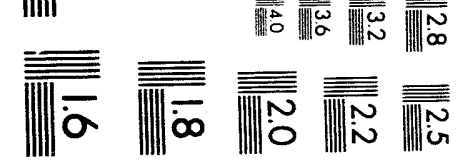



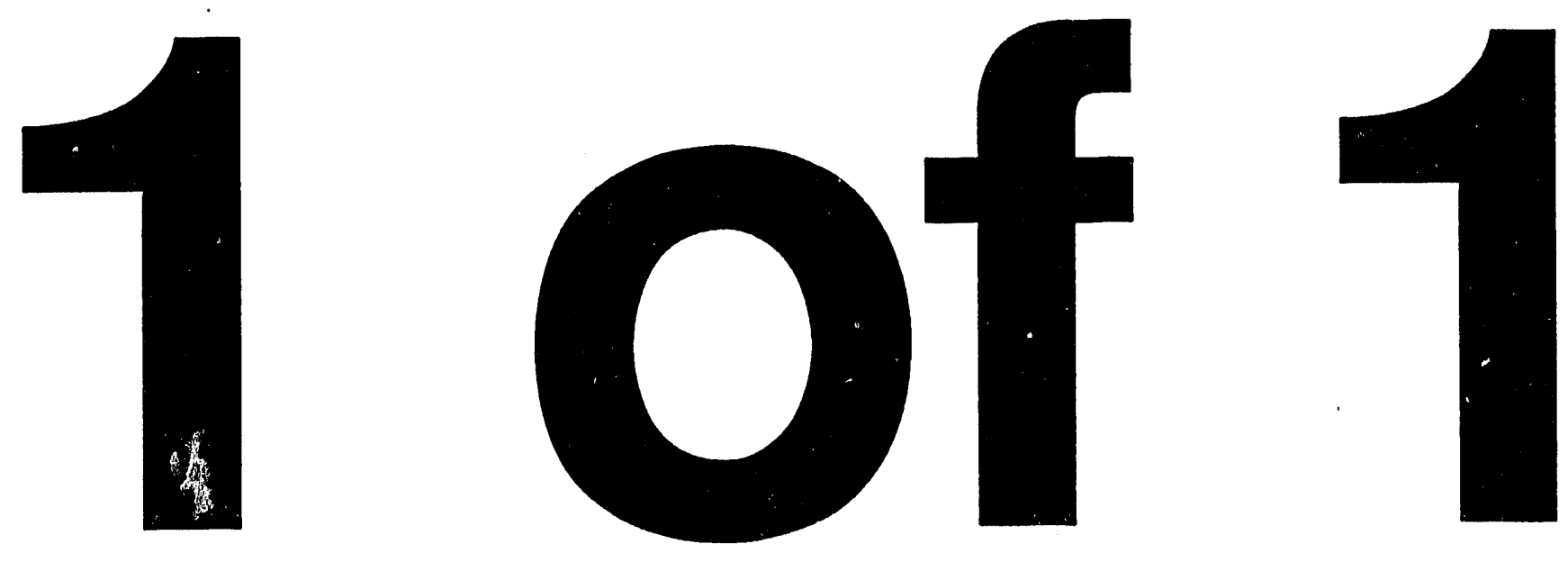
LBL-34015

CBP-026

UC-414

\title{
Generation of Harmonic Radiation \\ Using the Multi-Cavity Free-Electron Laser*
}

\author{
Srinivas Krishnagopal \\ Centre for Advanced Technology, Indore 452013, India \\ and \\ Andrew M. Sessler \\ Lawrence Berkeley Laboratory, University of California, Berkeley, California 94720
}

August 18, 1993

"Work supported in part by the Director, Office of Energy Research, Office of High Energy and Nuclear Physics, Division of High Energy Physics, of the U.S. Department of Energy under Contract No. DE-AC03-76SF00098 


\title{
Generation of Harmonic Radiation Using the Multi-Cavity
}

\section{Free-Electron Laser}

\author{
Srinivas Krishnagopal \\ Centre for Advanced Technology, Indore \$52013, India
}

\author{
Andrew M. Sessler \\ Lawrence Berkeley Laboratory, University of California, Berkeley CA 94720, USA \\ August 18, 1993
}

An FEL provides a convenient method of reaching short wavelengths by resonating with an input source at the fundamental wavelength while providing bunching at a harmonic of the fundamental. Recently schemes have been proposed that use two wiggler segments, one resonant at the fundamental to pre-bunch the beam, the other lasing at the desired (third) harmonic. A similar effect, with some advantages and some disadvantages, can be achieved using the Multi-Cavity FEL (MC/FEL). The MC/FEL employs several short cavities, operating in an oscillator-like manner, to achieve high output power. In this paper we consider the use of the MC/FEL as a means of generating harmonics. We investigate the competitiveness of this option in comparison with other harmonic generation schemes, in terms of the total wiggler length needed, the saturated power achieved, and the restrictions imposed by mirror reflectivity. 


\section{INTRODUCTION}

The essentially nonlinear nature of the FEL interaction makes it possible for electrons to radiate, on-axis, at odd harmonics of the fundamental frequency that is determined by the resonance condition. Operation in this mode is of interest because it allows one to push towards shorter wavelengths, and it has already been demonstrated experimentally $[1,2]$. The disadvantage of this approach lies in the fact that the comparatively weak harmonic signal has to be detected and extracted in the presence of the much stronger fundamental signal.

Another approach to reaching shorter wavelengths is that of the so-called Coherent Harmonic Generation in two-stage devices[3,4]. Here one takes advantage of the fact that the bunching of the electrons has all higher harmonic frequencies. An electron beam is run through an optical klystron-like set-up, where the first wiggler section defines a fundamental frequency $f_{R}$ and acts to bunch the beam. The second wiggler section, however, is designed for a harmonic frequency, say $3 f_{R}$. The electrons come pre-bunched into this section, and may therefore be expected to radiate copiously. An experiment using this scheme is to be implemented at Brookhaven $[5,6]$.

In this paper we consider a related approach, using the Multi-cavity Free-electron Laser (MC/FEL) that we have discussed earlier[7,8]. In the next section we review the MC/FEL and explain how we plan to generate coherent harmonic radiation using it. In Section 3 we present the results of one-dimensional numerical simulations to show that harmonic generation is indeed possible at infrared wavelengths. In Section 4 we consider the viability of cperation in the ultraviolet regime, where mirror reflectivities are low. We end with a discussion of the viability of this scheme and on future directions.

\section{THE CONCEPT}

The MC/FEL consists of a number of short optical cavities, each with a length less 
than the slippage distance between the electrons and the radiation[7]. Electrons are allowed to pass from cavity to cavity through a small hole drilled at the centre; however the radiation in a given cavity largely remains trapped within that cavity. Radiation is extracted only from the last cavity. Because the cavity is short, the length of the optical pulse is small; because the electron beam enters the last cavity substantially bunched, it can radiate appreciably. Thus the $\mathrm{MC} / \mathrm{FEL}$ produces brief, intense pulses of radiation.

For the present application, the cavities are divided into two sets. In the first set of cavities, which we call the fundamental cavities, the electrons radiate at a fundamental frequency $f_{R}$, and at the same time get bunched. These bunched electrons then enter the second set of cavities, the harmonic cavities, for which the wiggler wavelength (or field) is adjusted to make the resonant frequency $3 f_{R}$. Because the bunching of the electrons also has a component at this frequency, for this set of cavities they come pre-bunched, and therefore radiate quite substartially at the frequency $3 f_{R}$. As before, radiation is extracted only from the last cavity.

Clearly, this scheme operates the FEL in the oscillator mode, as opposed to the amplifier configuration of Ref.[3]. The advantage we gain here, besides the original one of getting short pulses, is of having a lesser length of wiggler since, as we will show, a few short wiggler segments are all we need. The disadvantage, on the other hand, is that at very short wavelengths, where mirror reflectivities are low, this scheme may be difficult to implement.

\section{OPERATION AT INFRARED WAVELENGTHS}

We employed the one-dimensional, time-independent FEL code described in Ref. [7] with suitable modifications. In each cavity the motion was described by the usual nonlinear equations which, in the noticion of Bonifacio, Pellegrini and Narducci[9] are: 


$$
\begin{aligned}
\left(\frac{d \theta_{j}}{d t}\right) & =\omega_{0}\left(1-\gamma_{R}^{2} / \gamma_{i}^{2}\right)+\frac{1}{2 \gamma_{i}^{2} \lambda}[J J]\left[\alpha \exp \left(i \theta_{j}\right)+c . c .\right] \\
\left(\frac{d \gamma_{j}}{d t}\right) & =\frac{-e c \kappa}{2 m c^{2} \gamma_{j}}[J J]\left[\alpha \exp \left(-i \theta_{j}\right)+c . c .\right] \\
\frac{1}{c} \frac{d \alpha}{d t} & =2 \pi n_{0} \frac{\kappa}{\Sigma}\left\langle\frac{e^{-i \theta_{j}}}{\gamma_{j}}\right\rangle .
\end{aligned}
$$

Here $\alpha$ is the complex amplitude of the electric field, $\theta_{j}$ is the phase of the $j^{\text {th }}$ electron relative to the electromagnetic field, and $\gamma_{j}$ is its energy in units of $m c^{2}$. The wiggler frequency is $\omega_{0}$, the wiggler parameter is $\kappa$, and $\lambda$ is the wavelength of the radiation field. The resonant energy is $\gamma_{R}$, the electron density is $n_{0}$ and $\Sigma$ is the effective transverse cross-section of the beam, describing the overlap of the beam with the radiation. The average $\langle\ldots\rangle$ is carried out over all electrons in the bunch. $[J J]$ is the usual Bessel function factor that is unity for helical wigglers.

Lasing was allowed in two sets of cavities, the second set lasing at some harmonic of the first, defined through an input parameter $N_{h}$. This was achieved, in the code, by increasing the input wiggler wave-number and the radiation frequency by this factor (thus keeping the beam energy fixed). In addition, the lengths of the harmonic cavities were also reduced by the same factor, so that in every cavity the electron beam saw the same number of wiggler periods. It was confirmed that with $N_{h}=1$, the results were identical with the earlier code that allowed for only one set of cavities.

It should be emphasized here that within each cavity the process of radiation generation is described by Eqs. 1-3, and is therefore at a single frequency. The term 'harmonic' is applied to the second set of cavities only because they are designed to lase at a frequency that is a multiple of the frequency in the first set of cavities. This usage is justified because it is the existence of harmonics in the bunching of the electrons that makes this scheme possible.

Parameters used in the results reported here are given in Table 1. In addition, all the 
mirrors were assumed to have a reflectivity of $R=0.98$, except for the last, out-coupling mirror, for which $R=0.95$. Eight hundred test particles were used and 40 longitudinal integrations steps were performed. Figure la shows plots of the power and bunching in the last harmonic cavity, i.e. the last overall cavity, as a function of bunch number. We see that the power saturates at around $10 \mathrm{MW}$, and that the bunching factor is large, though the electrons are not yet over-bunched. Note that this power level is achieved with a total wiggler length of only $40 \mathrm{~cm}$ !

The reason for this impressive performance has, of course, to do with the fact that the electrons entering the harmonic savities, designed for a resonant wavelength of $3 f_{R}$, have been pre-bunched. The fact that this pre-bunching is achieved at a lower frequency is what gives life to this option, because the small-signal gain is higher at lower frequencies. Thus, by using two different sets of cavities, we are able to run the first set at a lower trequency in order to take advantage of the larger small-signal gain. In the second set, though the gain is smaller, the electrons enter pre-bunched; this makes the effective length of the second set of cavities greater.

To demonstrate that this is indeed the case, Figure $1 \mathrm{~b}$ shows the power and bunching in the last (sixth) cavity when all cavities are run at the higher frequency. Essentially, we are now pre-bunching the beam directly at the higher frequency, and we can see that the power level attained is much lower.

\section{OPERATION AT ULTRAVIOLET WAVELENGTHS}

The more exciting application for schemes of coherent harmonic generation is to push back the short-wavelength frontier. The shortest wavelength at which lasing has been achieved in a free-electron laser is at $240 \mathrm{~nm}$, at the storage-ring FEL at Novosibirsk[10]. Clearly, it is of considerable interest to use the MC/FEL to lase at some harmonic of this frequency - say the third, i.e. at $80 \mathrm{~nm}$. 
The main problem in this effort is that as the wavelength decreases, mirror reflectivities decrease dramatically, making oscillator schemes unviable. Still, it seems that at $80 \mathrm{~nm}$, using a clean aluminum surface placed in ultra-high vacuum, it may be possible to achieve reflectivities of $R=0.80$ [11]. Using this number, we therefore attempted to simulate such a system, along the lines of the work in Ref. [3]. For most purposes, therefore, we stuck to their parameters; the full list of parameters is given in Table 2. The fundamental cavities were assumed to have a reflectivity of $R=0.95$ (which is a realistic number), and the harmonic cavities of $R=0.80$, except for the out-coupling mirror, for which $R=0.75$ was assumed.

Figure 2 summarizes the results in terms of the power and the bunching factor in the last cavity. It can be seen that the power in the cavity saturates at about $50 \mathrm{MW}$, which is considerable. Note that this is achieved with a total wiggler length of only $(3 \times 2.4)+(3 \times 0.8)=9.6 \mathrm{~m}$. We also checked that lasing at the harmonic continued to occur as the reflectivity was reduced from 0.80 down to around 0.65 . We thus conclude that the MC/FEL harmonic generation scheme is viable even at short wavelengths.

\section{DISCUSSION}

In the preceding sections we have shown that one can generate harmonic radiation using the MC/FEL. A few points need emphasis.

First, unlike the scheme of Ref [3] which operates the FEL in the amplifier configuration, this scheme operates it as an oscillator. The advantage we gain by this is that, since the power can be built up slowly over several passes, the gain within a cavity need only be modest, and consequently the wiggler segments can be short. Further, because the electron beam is considerably pre-bunched before entering the last wiggler, it can be expected to radiate substantially, even with a comparatively short length of wiggler. This makes our scheme economically more viable. 
Another advantage in our scheme is that frequency selection is not a problem, because the fundamental and 'harmonic' radiation are in different cavities. An incidental advantage is that one could perhaps get radiation at both frequencies, $\int_{R}$ and $3 f_{R}$, by simply arranging to extract the radiation from the last fundamental cav.ty also.

Further, since the harmonics are in the bunching of the electrons, and not in the frequency of the emitted radiation, it is possible to lase at all harmonics of the fundamental, and not at just the odd harmonics. Lasing occurs at all harmonics; the higher the harmonic number the lesser the saturated power, and the dependence is roughly exponential. Specifically, using parameters for the fundamental cavities from Table 1, we find that the saturated power in the last harmonic cavity reduces from $40 M W$ for the second harmonic to $5 M W$ for the fourth harmonic.

The major drawback, of course, is that the need to have mirrors limits the usefulness of this scheme at very short wavelengths. Nonetheless, as we have shown in Section 4, with present mirror technology the scheme is still viable down to around $80 \mathrm{~nm}$ (see Ref. [11] for details). For even shorter wavelengths it may be possible to employ a hybrid scheme in which one employs a MC/FEL at longer wavelengths, to pre-bunch the electron beam efficiently, and a long amplifier section to generate coherent radiation at the shorter wavelength.

\section{CONCLUSION}

We have shown, using one-dimensional simulations, that the MC/FEL can viably be used to produce harmonic radiation at both, infrared and ultraviolet wavelengths. The power levels achieved are substantial, and the total length of wiggler required is relatively small. The scheme warrants further study, especially of the mode structure of the electromagnetic beam, using three-dimensional simulations. 


\section{ACKNOWLEDGEMENTS}

This research was supported in part by the Director, Office of Energy Research, Office of High Energy and Nuclear Physics, Division of Higher Energy Physics, of the U.S. Department of Energy under contract DE-AC03-76SF00098. 


\section{REFERENCES}

[1] D. J. Bramford and D. A. G. Deacon, Nucl. Instr. and Meth. A296 (1990) 89.

[2] R. W. Warren, et al., Nucl. Instr, and Meth. A296 (1990) 84.

[3] R. Bonifacio, L. De Salvo Souza, P. Pierini and E. T. Scharlemann, Nucl. Instr. and Meth. A296 (1990) 787.

[4] R. Prazeres and J. M. Ortega, Nucl. Instr. and Meth. A296 (1990) 436.

[5] I. Ben-Zvi et al., Nucl. Instr. and Meth. A304 (1991) 72.

[6] L. H. Yu, Phys. Rev. A44 (1991) 5178.

[7] S. Krishnagopal, G. Rangarajan and A. M. Sessler, Opt. Commun. 100 (1993) 518.

[8] S. Krishnagopal, G. Rangarrijan and A. M. Sessler, Nucl. Instr. and Meth. A331 (1993) ABS 22.

[9] R. Bonifacio, C.Pelligrini and L. Narducci, Opt. Commun. 50 (1984) 373.

[10] G.A.Kornyukhin et al., Nucl. Instr. and Meth. A237 (1986) 281.

[11] Extreme Ultraviolet and Soft X-Ray Optics For Free Electron Lase: ^, J.B.Kortright, in Laser Handbook Vol 6, edited by W.B.Colson, C.Pellegrini and A.Renieri, Eisevier Science Publishers B.V. (1990) p. 463. 


\section{TABLES}

TABLE I. Parameters used in the simulations of Section 3 (infrared wavelengths).

\begin{tabular}{|c|c|}
\hline Parameter & Value \\
\hline Wiggler parameter $\left(a_{w}\right)$ & 1.5 \\
\hline Current $(I)$ & $30 \mathrm{~A}$ \\
\hline Energy $\left(\gamma m c^{2}\right)$ & $7.5 \mathrm{MeV}$ \\
\hline Pulse length & $20 \mathrm{nsec}$ \\
\hline Initial optical power $\left(P_{i n}\right)$ & $100 \mathrm{~W}$ \\
\hline \multicolumn{2}{|l|}{ Fundamental Cavities } \\
\hline Number of cavities & 3 \\
\hline Wiggler length $(L)$ & $10 \mathrm{~cm}$ \\
\hline Wiggler period $\left(\lambda_{w}\right)$ & $2 \mathrm{~cm}$ \\
\hline Optical wavelength & $100 \mu \mathrm{m}$ \\
\hline Reflectivity $(R)$ & 0.98 \\
\hline \multicolumn{2}{|l|}{ Harmonic Cavities } \\
\hline Harmonic Number $\left(N_{h}\right)$ & 3 \\
\hline Number of cavities & 3 \\
\hline Wiggler length $(L)$ & $3.33 \mathrm{~cm}$ \\
\hline Wiggler period $\left(\lambda_{w}\right)$ & $0.667 \mathrm{~cm}$ \\
\hline Optical wavelength & $33.3 \mu \mathrm{m}$ \\
\hline Reflectivity $(R)$ & 0.98 \\
\hline Out-coupling Reflectivity $\left(R_{o u t}\right)$ & 0.95 \\
\hline
\end{tabular}


TABLE II. Parameters used in the simulations of Section 4 (ultraviolet wavelengths).

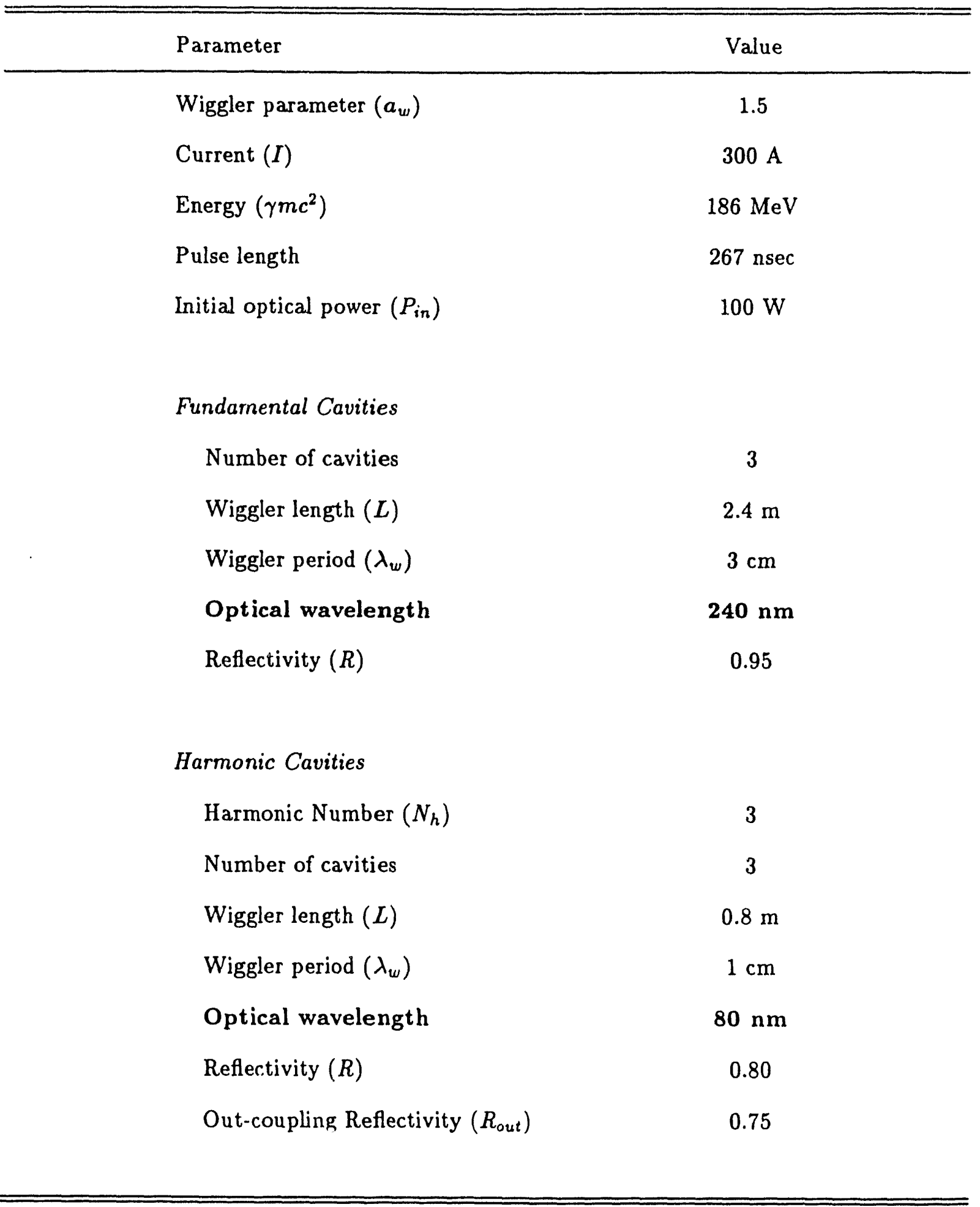




\section{FIGURES}

FIG. 1. Plots of power and bunching factor in the last (sixth) cavity, which lases at $33.3 \mu \mathrm{m}$, when the first three cavities pre-bunch the beam, (a) at $100 \mu \mathrm{m}$, and (b) directly at 33.3 $\mu m$. Details in Section 3.

FIG. 2. Plots of power and bunching factor in the last (sixth) cavity, which lases at $80 \mathrm{~nm}$, when the first three cavities pre-bunch tha beam at $240 \mathrm{~nm}$. Details in Section 4. 


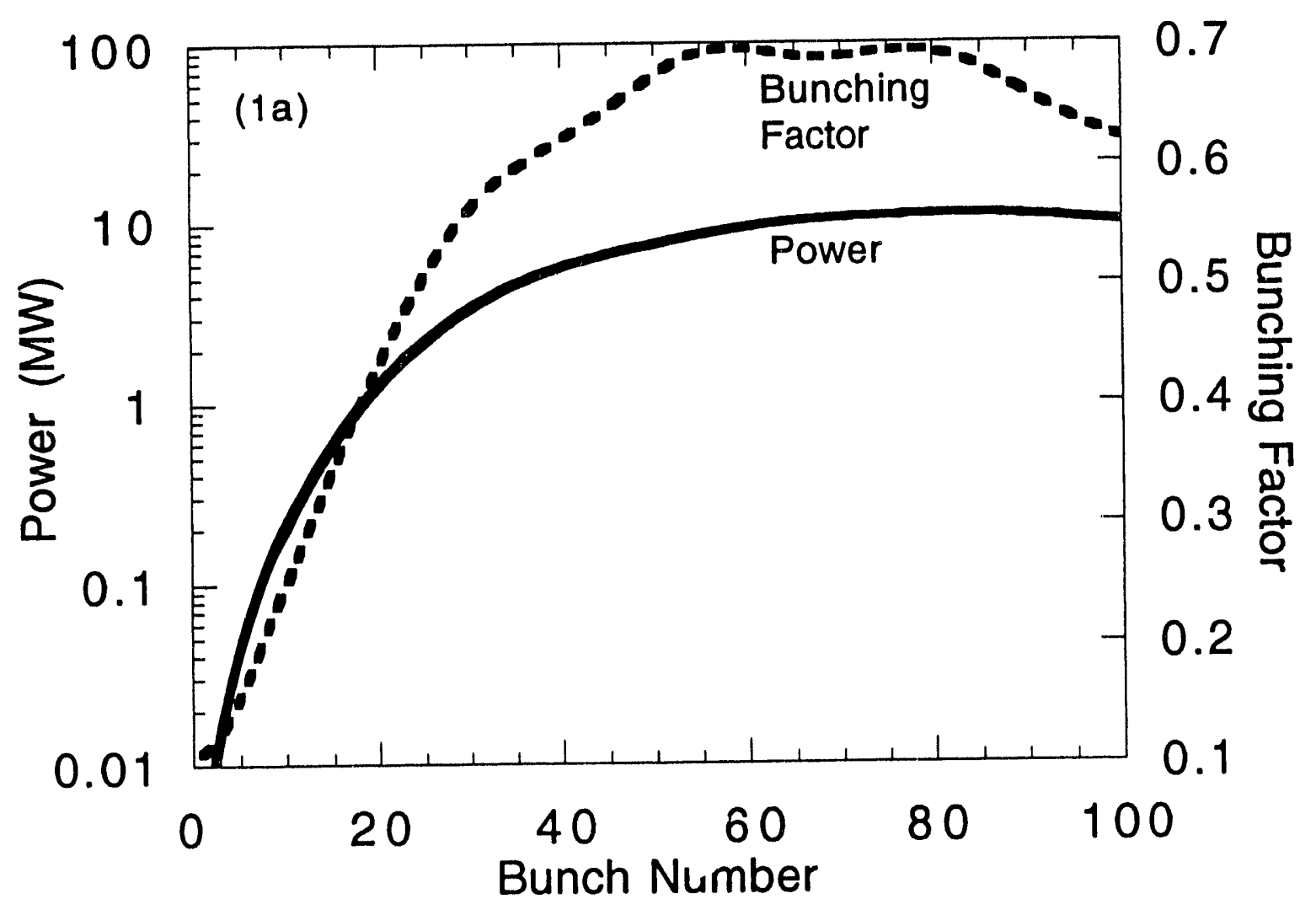

Fig. 1a 


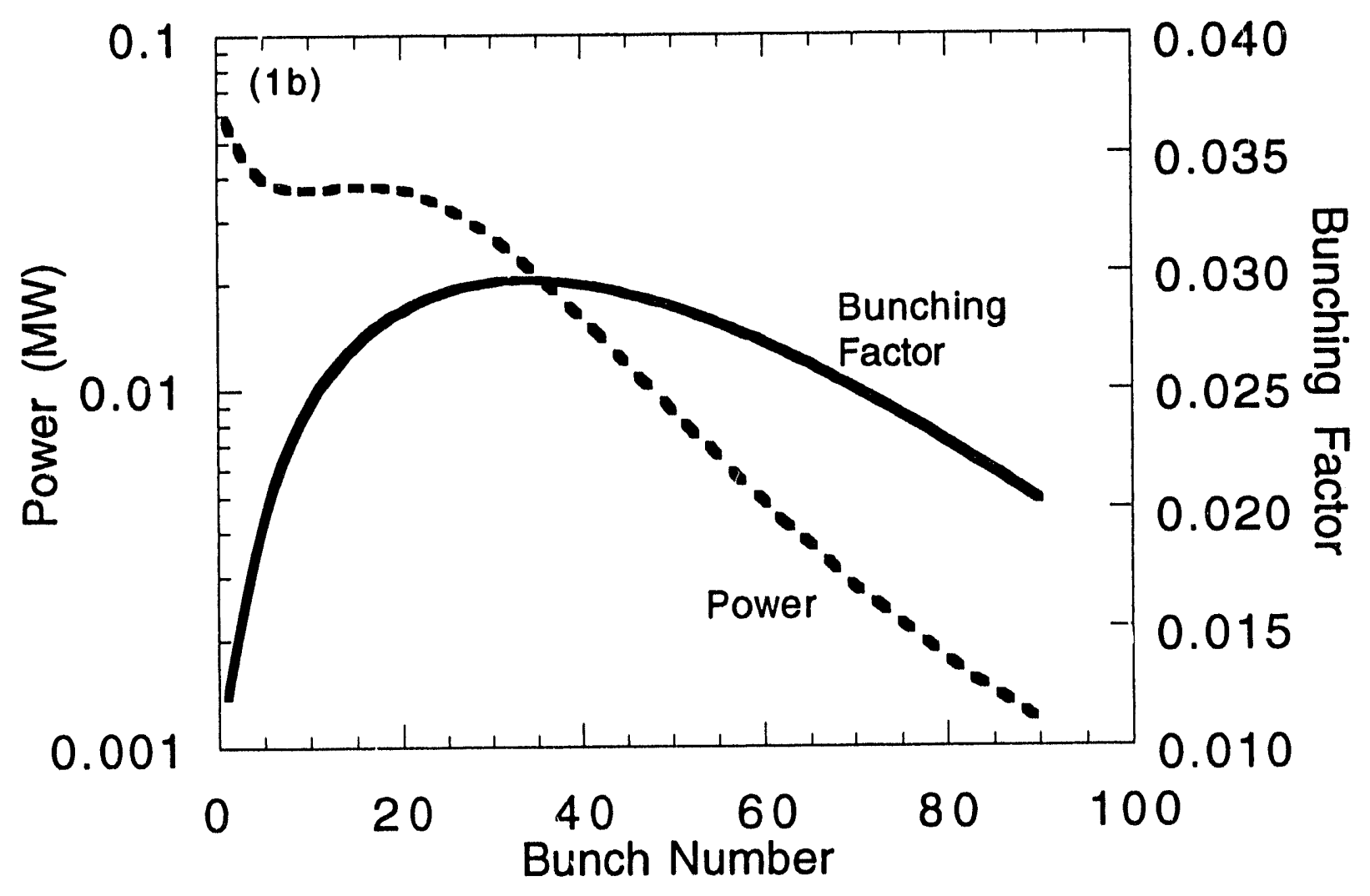

Fig. 1b 


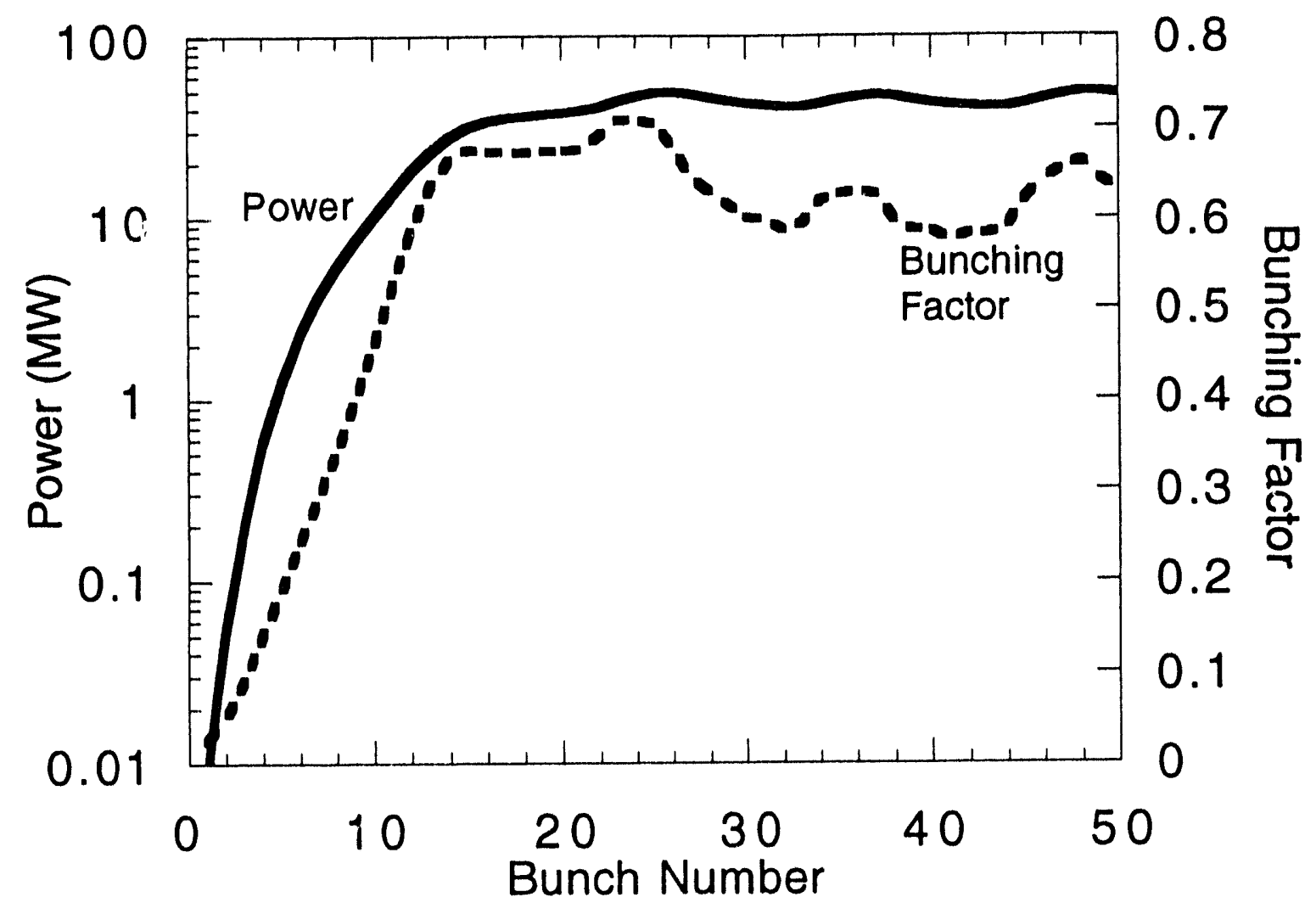

Fig. 2 

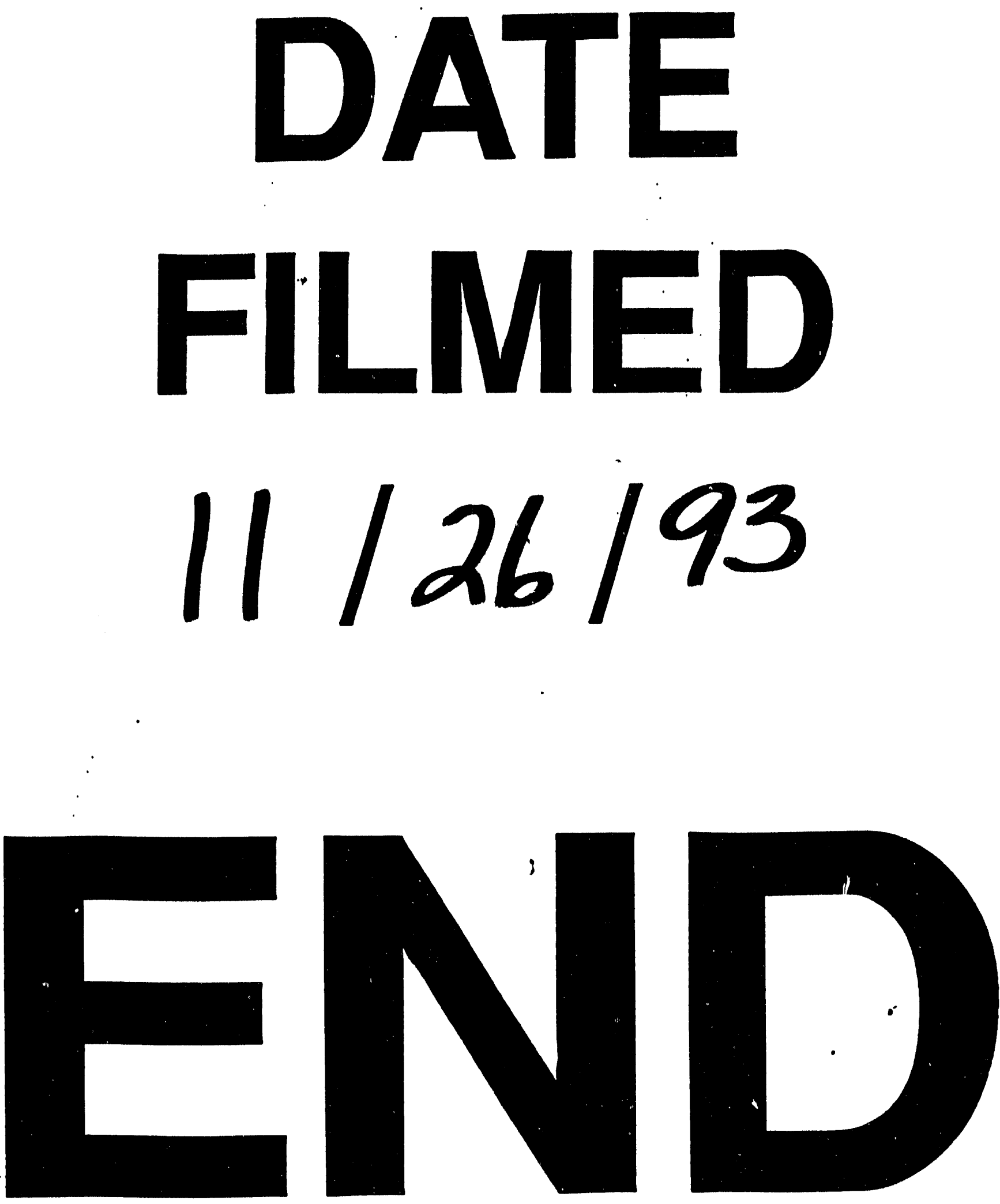
